\title{
Microbiome analysis of contact lens care solutions and tear fluids of contact lens wearers: Possible involvement of streptococcal antigens in allergic symptoms related to contact lens wear
}

\author{
FUMIKA HOTTA $^{1,2}$, HIROSHI EGUCHI ${ }^{1,2}$, HARUYUKI NAKAYAMA-IMAOHJI ${ }^{2}$, TOMOMI KUWAHARA ${ }^{2}$, \\ AYANO TADA $^{2}$, HIROFUMI YAGI ${ }^{2}$, YOSHIKAZU SHIMOMURA ${ }^{3}$ and SHUNJI KUSAKA ${ }^{1}$ \\ ${ }^{1}$ Department of Ophthalmology, Kindai University Faculty of Medicine, Osakasayama, Osaka 589-8511; \\ ${ }^{2}$ Department of Microbiology, Faculty of Medicine, Kagawa University, Miki, Kagawa 761-0793; \\ ${ }^{3}$ Department of Ophthalmology, Fuchu Eye Center, Izumi, Osaka 594-0076, Japan
}

Received February 12, 2020; Accepted June 24, 2020

DOI: $10.3892 / \mathrm{ijmm} .2020 .4678$

\begin{abstract}
The present study elucidated the pathogenesis of allergic symptoms (AS) related to contact lens (CL) wear by assaying CL care solutions in lens storage cases and tears from subjects with AS using molecular biology techniques. A total of 15 CL storage cases were collected from subjects with AS ( $n=9)$ and healthy, asymptomatic control CL wearers $(n=6)$. Bacterial populations in CL care solutions and tears were assayed by culture and 16S rDNA sequencing. Histamine levels in tears were measured by high-performance liquid chromatography. Western blot analysis was performed to identify the bacteria recognized by tear IgE from subjects with AS. No significant differences were found in the culture results between the subjects with AS and asymptomatic subjects. Histamine was detected in 2 subjects with AS. Meta-16S rDNA sequencing identified a cluster of 4 subjects with AS that were distinguished from others by principal coordinate analysis. Detailed population analysis revealed that the abundance of Gram-positive bacteria in the microbiomes of CL care solutions used by the subjects with AS were higher than those of asymptomatic subjects $(42.24 \pm 9.47$ vs. $16.85 \pm 22.76 \%$ abundance). Among these, Streptococcus was the dominant genus (12.1-18.3\% abundance). Tear microbiome
\end{abstract}

Correspondence to: Professor Hiroshi Eguchi, Department of Ophthalmology, Kindai University Faculty of Medicine, 377-2 Ohnohigashi, Osakasayama, Osaka 589-8511, Japan

E-mail: hiroegu0113@gmail.com

Abbreviations: AS, allergic symptoms; CL, contact lens; CLPC, contact lens-induced papillary conjunctivitis; NGS, next-generation sequencing; BHI, brain heart infusion; TE, Tris-ethylenediaminetetraacetic acid; HPLC, high-performance liquid chromatography; PBS, phosphate-buffered saline; OUT, operational taxonomic unit; PCoA, principal coordinate analysis; PFA, paraformaldehyde; PBS-T, PBS with Tween-20

Key words: microbiome, tear, contact lens care solution, Streptococcus, allergy analysis revealed that the abundance of Streptococcus in the subjects with AS was significantly higher than that in other subjects $(19.02 \pm 5.50$ vs. $3.08 \pm 3.35 \%, \mathrm{P}<0.01)$. Western blot analysis demonstrated that the tear $\operatorname{IgE}$ in all subjects with AS reacted with Streptococcus (100\%), but not with Staphylococcus. On the whole, these results provide novel insight into the pathogenesis of AS and identify Streptococcus as an important factor in AS associated with CL wear.

\section{Introduction}

Contact lens (CL)-induced papillary conjunctivitis (CLPC) is a chronic conjunctivitis that occurs in CL wearers. Physical stimulation to the palpebral conjunctiva by the CL itself and allergic reactions to substances adhering to the CL surface are considered among the primary causes (1). Although the types of depositions that induce an allergic reaction have not yet been confirmed, bio-deposits of denatured protein are predicted to be one of the etiologies $(2,3)$. Bacteria adhering to the $\mathrm{CL}$ surface are possible allergens for CLPC. However, this has not been investigated in depth thus far. Tagliaferri et al cultivated CLs collected from patients with CLPC and reported that there was no association between CLPC and bacteria adhering to the CL surface (4). However, the authors of that study evaluated the association only by the use of conventional cultivation methods, and they did not analyze the bacterial population at the genus level. Since CL wear affects the ocular surface through various waterborne bacteria of environmental origin (5), and many of the environmental bacteria are fastidious (6), the validation of CL and care solutions in a culture-independent manner is desirable.

Recently, microbiome analysis employing next-generation sequencing (NGS) has been applied to gut, skin, oral, and vaginal samples (7-9). Several studies have analyzed the ocular surface microbiome of healthy subjects with NGS (10-12). These studies report that the composition of the ocular surface microbiome inferred by NGS greatly differs from that previously described using conventional culture methods $(10,12)$.

It is known that microbiomes are present in human organs, where they contribute to maintaining the homeostasis of each organ and/or human health. For example, in a study on the 
gut microbiome, the alteration of the gut microbiome termed dysbiosis was found to be involved in the development of non-infectious diseases, such as obesity, diabetes mellitus, autoimmune disease and inflammatory bowel disease $(13,14)$. Similarly, the ocular surface microbiome may play an important role in ocular surface homeostasis, and its alteration may be related to the onset and deterioration of some non-infectious eye diseases. Thus, micobiome analysis using NGS of ocular surface and ophthalmic clinical samples may help to elucidate the causal association between microorganism and non-infectious ocular diseases.

In the present study, NGS analysis of the microbiome in CL care solutions remaining in the lens storage cases and the tear fluids of CL wearers with allergic symptoms (AS) was performed. The findings of the present study suggest a possible association between bacterial contamination in the lens storage case and AS.

\section{Materials and methods}

Participant profiles. In total, $15 \mathrm{CL}$ wearers (9 subjects with AS and 6 healthy controls, ranging in age from 19 to 24 years) were enrolled in the present study (Table I). The present study followed the tenets of the Declaration of Helsinki and was approved by the Institutional Review Board of Kindai University Faculty of Medicine (approval no. 28-137). Informed consent was obtained from all subjects after explanation of the nature and possible consequences of the study. They were all volunteers who wore refractive soft hydrogel lenses, use multi-purpose solution for their lens care, and answered the questions on AS listed below (regarding the symptoms) after providing the lens storage case. Although all the brand names of lenses and care solutions could not be ascertained, the care solutions enrolled in the present study contained polidronium chloride or polyhexamethylene biguanide and all lenses were made of silicone hydrogel. The diagnostic criteria of AS include soft CL wearers who have symptoms of eye itching plus at least one of the following symptoms during CL wear: Conjunctival hyperaemia, eye discharge, foreign body sensation, or eye discomfort. Healthy subjects were defined as soft CL wearers who did not have any of the above-mentioned symptoms. Individuals who were administered any topical medications of anti-allergic agents and/or antimicrobial agents within 3 months prior to initiation of the present study were excluded. Individuals diagnosed with dry eye or atopic dermatitis were excluded.

Isolation of contaminated bacteria from CL care solution. The CL care solutions remaining in the CL storage cases were collected within $4 \mathrm{~h}$ of CL wear on the same day. The solution was sampled $(0.1 \mathrm{ml})$ and spread onto a brain heart infusion (BHI; Eiken Chemical Co., Ltd.) agar plate. The plate was incubated at $37^{\circ} \mathrm{C}$ for $48 \mathrm{~h}$. The colonies formed on the plate were individually inoculated into $\mathrm{BHI}$ broth. The culture was centrifuged $\left(10,000 \times \mathrm{g}, 5 \mathrm{~min}, 4^{\circ} \mathrm{C}\right)$, washed once with Tris-ethylenediaminetetraacetic acid (TE) buffer $[10 \mathrm{mM}$ Tris- $\mathrm{HCl}$ ( $\mathrm{pH} 8.0), 1 \mathrm{mM}$ ethylenediaminetetraacetic acid] and resuspended in $0.5 \mathrm{ml}$ of TE buffer. Using a part of the suspension, Gram staining was performed. After boiling the samples, they were centrifuged $\left(10,000 \times \mathrm{g}, 5 \mathrm{~min}, 4^{\circ} \mathrm{C}\right)$, and the supernatants were used as templates for $16 \mathrm{~S}$ rDNA amplification using universal primers 27F (5'-AGAGTTTGATCM TGGCTCAG-3') and 1492R (5'-GGMTACCTTGTTACG ACTT-3'). Species identification was performed by a BLAST (https://blast.ncbi.nlm.nih.gov/Blast.cgi) search of the obtained $16 \mathrm{~S}$ rDNA sequences against the $16 \mathrm{~S}$ ribosomal RNA database in NCBI. The species of each colony was identified as the best-hit microorganism (>97\% identity) by a BLAST search.

Quantification of histamine by high-performance liquid chromatography (HPLC). Tear fluids were collected from the study subjects during CL wearing at the same time when their CL care solutions were collected by soaking in Schirmer test paper (Eagle Vision) and distillation in phosphate-buffered saline (PBS). An additional experiment using saline solution to measure the amount of tear fluids per $1 \mathrm{~mm}$ of Schirmer test paper revealed that the paper was soaked with approximately $0.5 \mu \mathrm{l}$ of fluids per $1 \mathrm{~mm}$ (Table SI). Therefore, the amount of tear fluids collected from the subjects was approximately $5 \mu 1$, since they were collected when the scale of the Schirmer test paper reached $10 \mathrm{~mm}$. For 8 subjects with a tear-soaked scale of $\geq 15 \mathrm{~mm}$ on the Schirmer test paper, $0.5 \mu 1$ the tear fluids were used for western blot analysis as described below. The tear fluids of the subjects with AS were collected during the symptomatic period. Each diluted tear fluids $(0.1 \mathrm{ml})$ was mixed with $0.3 \mathrm{ml}$ of $0.6 \mathrm{M} \mathrm{HClO}_{4}$. After vortexing, the mixture was centrifuged for $15 \mathrm{~min}$ at $10,000 \mathrm{xg}$. The supernatant was filtered through a $0.2-\mu \mathrm{m}$ membrane. An aliquot of $80 \mu \mathrm{l}$ of the extract was used for derivatization. The extract samples containing $0.8 \mu \mathrm{g} / \mathrm{ml}$ of 1,7-diaminoheptane as an internal standard were dansylated as previously described by Chiacchierini et al (15). The concentration of dansylated histamine was determined by HPLC. HPLC was performed with a Tosoh 8020 HPLC system equipped with a CCPM-II pump, a UV-8020 variable-wavelength detector, a CO-8020 column oven, and a Degasys DG-1210 in-line degasser (Uniflows Co., Ltd.). The chromatographic peak area was computed with a SIC chromatorecorder (System Instruments Co., Ltd.). The separation was performed on a reverse phase TSKgel ODS-80Tm column (4.6x150 mm, Tosoh Corporation). The column temperature was maintained at $25^{\circ} \mathrm{C}$, and absorbance was monitored at $254 \mathrm{~nm}$. The $50 \mu \mathrm{l}$ each of the samples was injected into the column and eluted at a flow rate of $1.2 \mathrm{ml} / \mathrm{min}$. The mobile phase was acetonitrile- $\mathrm{H}_{2} \mathrm{O}(60: 40$, v/v). Histamine hydrochloride (Sigma-Aldrich; Merck KGaA) was used as a standard.

Microbiome analysis. Tear fluids and CL care solutions were centrifuged $\left(10,000 \mathrm{x} \mathrm{g}, 5 \mathrm{~min}, 4^{\circ} \mathrm{C}\right)$, and the pellets were washed once with TE buffer ( $\mathrm{pH} 8.0)$. Following centrifugation $\left(10,000 \mathrm{x} \mathrm{g}, 5 \mathrm{~min}, 4^{\circ} \mathrm{C}\right)$, the pellets were frozen at $-80^{\circ} \mathrm{C}$ until use. DNA extraction was performed as per the method previously reported by Morita et al (16). Sequencing libraries were prepared by amplifying the V3-V4 region of the $16 \mathrm{~S}$ rDNA using the primers (5'-TCGTCGGCAGCGTCAGAT GTGTATAAGAGACAGCCTACGGGNGGCWGCAG-3' and 5'-TCGTCGGCAGCGTCAGATGTGTATAAGAGACAG CCTACGGGNGGCWGCAG-3' and 5'-GTCTCGTGGGCT CGGAGATGTGTATAAGAGACAGGACTACHVGGGTAT CTAATCC-3') previously described by Klindworth et al (17). Following initial amplification, a second polymerase chain 
Table I. Sample profiles and the results of bacterial culture and histamine quantification.

\begin{tabular}{|c|c|c|c|c|c|c|}
\hline \multirow[b]{2}{*}{$\begin{array}{l}\text { Subject } \\
\text { ID }\end{array}$} & \multirow[b]{2}{*}{ Age, sex } & \multirow[b]{2}{*}{$\begin{array}{c}\text { Ocular } \\
\text { status }\end{array}$} & \multirow[b]{2}{*}{$\begin{array}{c}\text { Sample } \\
\text { type }\end{array}$} & \multicolumn{2}{|r|}{ Bacterial culture } & \multirow[b]{2}{*}{$\begin{array}{l}\text { Histamine } \\
\quad(\mu \mathrm{g} / \mathrm{ml})\end{array}$} \\
\hline & & & & Total counts/ml & Isolated species & \\
\hline 1 & 24, Male & AS & $\begin{array}{l}\text { Tear } \\
\text { CL }\end{array}$ & $\begin{array}{c}\text { ND } \\
2,000\end{array}$ & $\begin{array}{l}\text { Streptococcus tigurinus } \\
\text { Chryseobacterium indologenes } \\
\text { Microbacterium maritypicum } \\
\text { Comamonas koreensis } \\
\text { Bacillus subtilis }\end{array}$ & $<0.01$ \\
\hline 2 & 21, Female & AS & $\begin{array}{l}\text { Tear } \\
\text { CL }\end{array}$ & $\begin{array}{l}\text { ND } \\
\text { ND }\end{array}$ & & 0.032 \\
\hline 3 & 20, Female & AS & $\begin{array}{l}\text { Tear } \\
\text { CL }\end{array}$ & $\begin{array}{c}\text { ND } \\
60\end{array}$ & $\begin{array}{l}\text { Bacillus subtilis } \\
\text { Pseudomonas marginalis }\end{array}$ & $<0.01$ \\
\hline 4 & 21, Female & AS & $\begin{array}{l}\text { Tear } \\
\text { CL }\end{array}$ & $\begin{array}{c}\text { ND } \\
60\end{array}$ & Pseudomonas marginalis & $<0.01$ \\
\hline 5 & 20, Female & AS & $\begin{array}{l}\text { Tear } \\
\text { CL }\end{array}$ & $\begin{array}{c}\text { ND } \\
841,000\end{array}$ & $\begin{array}{l}\text { Delftia tsuruhatensis } \\
\text { Pseudomonas hibiscicola } \\
\text { Microbacterium testaceum } \\
\text { Xenophilus aerolatus }\end{array}$ & $<0.01$ \\
\hline 6 & 20, Female & AS & $\begin{array}{l}\text { Tear } \\
\text { CL }\end{array}$ & $\begin{array}{l}\text { ND } \\
\text { ND }\end{array}$ & & $<0.01$ \\
\hline 7 & 19, Female & AS & $\begin{array}{l}\text { Tear } \\
\text { CL }\end{array}$ & $\begin{array}{l}\text { ND } \\
\text { ND }\end{array}$ & & 0.223 \\
\hline 8 & 21, Female & AS & $\begin{array}{l}\text { Tear } \\
\text { CL }\end{array}$ & $\begin{array}{l}\text { ND } \\
\text { ND }\end{array}$ & & $<0.01$ \\
\hline 9 & 21, Male & AS & $\begin{array}{l}\text { Tear } \\
\text { CL }\end{array}$ & $\begin{array}{c}\text { ND } \\
20\end{array}$ & Staphylococcus epidermidis & $<0.01$ \\
\hline 10 & 24, Male & Healthy & $\begin{array}{l}\text { Tear } \\
\text { CL }\end{array}$ & $\begin{array}{l}\text { ND } \\
\text { ND }\end{array}$ & & $<0.01$ \\
\hline 11 & 20 , Female & Healthy & $\begin{array}{l}\text { Tear } \\
\text { CL }\end{array}$ & $\begin{array}{l}\text { ND } \\
\text { ND }\end{array}$ & & $<0.01$ \\
\hline 12 & 21, Female & Healthy & $\begin{array}{l}\text { Tear } \\
\text { CL }\end{array}$ & $\begin{array}{l}\text { ND } \\
\text { ND }\end{array}$ & & $<0.01$ \\
\hline 13 & 23, Female & Healthy & $\begin{array}{l}\text { Tear } \\
\text { CL }\end{array}$ & $\begin{array}{c}\text { ND } \\
521,000\end{array}$ & $\begin{array}{l}\text { Microbacterium trichothecenolyticum } \\
\text { Pseudomonas azotoformans } \\
\text { Microbacterium testaceum }\end{array}$ & $<0.01$ \\
\hline 14 & 21, Female & Healthy & $\begin{array}{l}\text { Tear } \\
\text { CL }\end{array}$ & $\begin{array}{c}\text { ND } \\
14,900\end{array}$ & Pantoea vagans & $<0.01$ \\
\hline 15 & 21, Female & Healthy & $\begin{array}{l}\text { Tear } \\
\text { CL }\end{array}$ & $\begin{array}{l}200 \\
\text { ND }\end{array}$ & Staphylococcus capitis & $<0.01$ \\
\hline
\end{tabular}

AS, allergic symptoms; CL, contact lens care solution; ND, not detected.

reaction was performed with Nextra XT Index kit (cat. no. 15055294, Illumina, Inc.) to attach Illumina adaptors, as well as barcodes that allow for multiplexing. Amplification was performed in $25 \mu \mathrm{l}$ reactions containing $2.5 \mu \mathrm{l}$ of diluted template, $12.5 \mu \mathrm{l}$ of 2X KAPA HiFi HotStart Ready
Mix (cat. no. KK2602, Kapa Biosystems), and $5 \mu$ l each of primers. Thermal cycling consisted of an initial denaturation step $\left(3 \mathrm{~min}\right.$ at $95^{\circ} \mathrm{C}$ ), followed by 25 cycles of denaturation $\left(30 \mathrm{sec}\right.$ at $\left.95^{\circ} \mathrm{C}\right)$, annealing $\left(30 \mathrm{sec}\right.$ at $\left.55^{\circ} \mathrm{C}\right)$, and $30 \mathrm{sec}$ extension at $72^{\circ} \mathrm{C}$. The final extension was performed for $5 \mathrm{~min}$ 
at $72^{\circ} \mathrm{C}$. Amplicons were purified using AMPure XP beads (cat. no. A63881, Beckman Coulter, Inc.). The integrity of the DNA amplicon library was evaluated by an Agilent 2100 Bioanalyzer (Agilent Technologies Japan, Ltd.) using an Agilent High Sensitivity DNA kit (cat. no. 5067-4626, Agilent Technologies Japan, Ltd.). The loading DNA concentration was adjusted to $6 \mathrm{pM}$ each with Qubit Fluorometer Q32857 (Thermo Fisher Scientific K.K.) using a Qubit dsDNA BR Assay kit (cat. no. Q32853, Thermo Fisher Scientific K.K.). Sequencing was performed on the Illumina MiSeq platform (cat. no. MS-102-3003, MiSeq Reagent kit version 3,600 cycles, Illumina, Inc.) according to the manufacturer's specifications to generate paired-end reads of 300 bases in length in each direction. In total, 15,992,047 2×300 base pair reads with an average of 333,168 reads per sample was obtained. Primer sequences were trimmed away, and the paired-end reads were merged using fastq-join (18) with default parameters and processed with the QIIME 1.8.0 pipeline (19). Following a chimera check by Usearch v6.1.544 (20), 20,000 Illumina reads per sample (average Phred $>20$ ) were randomly selected for further analysis. Using the UCLUST (20) algorithm built into the QIIME pipeline, sequences were clustered at $97 \%$ identity against the Greengenes reference database (http://greengenes. lbl.gov), producing 744 operational taxonomic units (OTUs). Using the QIIME pipeline, unweighted UniFrac distances were produced and used for investigation of beta diversity through plotting principal coordinate analysis (PCoA) coordinates. QIIME analysis pipeline that include these software was downloaded through QIIME (http://qiime.org/).

Western blot analysis against ocular bacteria by tear IgE. Staphylococcus aureus 209P, Staphylococcus epidermidis (isolated from the CL care solution of subject 9), Staphylococcus capitis (isolated from the tear fluid of subject 15), Streptococcus pneumoniae IID555 and Streptococcus tigurinus (isolated from the CL care solution of subject 1) were cultured under microaerophilic conditions $\left(5 \% \mathrm{CO}_{2}\right)$ in $\mathrm{BHI}$ at $37^{\circ} \mathrm{C}$ for $24 \mathrm{~h}$. The bacterial cells were centrifuged $(10,000 \mathrm{x} \mathrm{g}$, $5 \mathrm{~min}, 4^{\circ} \mathrm{C}$ ), washed once with PBS, and resuspended in $4 \%$ paraformaldehyde in PBS (PFA) to $\mathrm{OD}_{600}=1.0$. The culture supernatants were mixed with PFA at 1:1. The $2 \mu \mathrm{l}$ each of the bacterial cell suspension, culture supernatants, or purified human $\operatorname{IgE}(\mathrm{H}+\mathrm{L} ; 1.0 \mu \mathrm{g} / \mathrm{ml}$, cat. no. ab65866, Abcam) were plotted onto a nitrocellulose membrane and dried overnight at $25^{\circ} \mathrm{C}$. Blocking was performed with $3 \%$ skim milk in PBS with Tween-20 (PBS-T) for $1 \mathrm{~h}$ at $25^{\circ} \mathrm{C}$. The membrane was washed 3 times with PBS-T. The membrane was incubated with diluted tear fluids (10 $\mu \mathrm{l} \mathrm{each)} \mathrm{in} 3 \mathrm{ml}$ of PBS-T at $30^{\circ} \mathrm{C}$ for $1 \mathrm{~h}$. After washing 3 times with PBS-T, goat-anti human $\operatorname{IgE}$ (H+L) HRP-conjugated (1:1,000) (cat. no. ab73901, Abcam) was added and incubated at $30^{\circ} \mathrm{C}$ for $1 \mathrm{~h}$ with gentle agitation. The membrane was washed 3 times with PBS-T, and the chemiluminescence signal developed by ECL Prime Western Blotting Detection Reagent (GE Healthcare UK Ltd.) was detected with LAS4000 (GE Healthcare UK Ltd.). To examine the non-specific binding of $\operatorname{IgE}$ to the bacterial cells, human $\operatorname{IgE}(1.0 \mu \mathrm{g} / \mathrm{ml})$ was used instead of tear fluids. Western dot-blot hybridization was applied to 6 subjects with AS and 2 healthy subjects who were able to collect enough tear fluids for the examination.
Statistical analysis. The minimum sample size required for the present study was calculated using Easy R (EZR) version 1.41 (21). Quantitative microbiological measurements were transformed to $\log _{10}$ prior to conducting an analysis. All experiments were performed at least 3 times. The data were analysed by Chi-squared test, one-way analysis of variance and Tukey's post hoc test (StatFlex ver.6, Artec Co., Ltd., Tokyo, Japan) to assess the significance. P-values $<0.05$ were considered to indicate statistically significant differences.

Sequence data deposition. Sequence data have been deposited in the DDBJ database (accession nos. DRA010383: https://dbj.nig.ac.jp/DRASearch/submission?acc=DRA010383; PRJDB10068: https://ddbj.nig.ac.jp/BPSearch/bioproject? acc=PRJDB10068; SAMD00232908-SAMD00232922: https://ddbj.nig.ac.jp/DRASearch/experiment?acc=DRX224997, https://ddbj.nig.ac.jp/DRASearch/experiment?acc=DRX224998, https://ddbj.nig.ac.jp/DRASearch/experiment?acc=DRX224999, https://ddbj.nig.ac.jp/DRASearch/experiment?acc=DRX225000, https://ddbj.nig.ac.jp/DRASearch/experiment?acc=DRX225001, https://ddbj.nig.ac.jp/DRASearch/experiment?acc=DRX225002, https://ddbj.nig.ac.jp/DRASearch/experiment?acc=DRX225003, https://ddbj.nig.ac.jp/DRASearch/experiment?acc=DRX225004, https://ddbj.nig.ac.jp/DRASearch/experiment?acc=DRX225005, https://ddbj.nig.ac.jp/DRASearch/experiment?acc=DRX225006, https://ddbj.nig.ac.jp/DRASearch/experiment?acc=DRX225007, https://ddbj.nig.ac.jp/DRASearch/experiment?acc=DRX225008, https://ddbj.nig.ac.jp/DRASearch/experiment?acc=DRX225009, https://ddbj.nig.ac.jp/DRASearch/experiment?acc=DRX225010, https://ddbj.nig.ac.jp/DRASearch/experiment?acc=DRX225011).

\section{Results}

Sample collection, isolation of bacteria and histamine level in tear fluids. Tear fluids and CL care solutions remaining in lens storage cases were collected from $15 \mathrm{CL}$ wearers $(9$ subjects with AS and 6 healthy subjects) (Table I). Bacterial cultures of CL care solutions were positive in 5 of the 9 subjects with AS and in 2 of the 6 healthy subjects, and diverse bacteria were detected. The culture positive ratios between CL care solutions from subjects with AS and healthy subjects did not exhibit any statistically significant differences $(\mathrm{P}=0.3980$, data not shown). All the tear cultures were negative apart from 1 participant (subject 15) from whom the Staphylococcus capitis was isolated. The total numbers of bacteria in culture positive CL care solutions did not differ significantly between the subjects with AS and healthy subjects $\left(\log _{10} \mathrm{CFU} / \mathrm{ml}, 2.82 \pm 1.89\right.$ vs. $4.95 \pm 1.09$, $\mathrm{P}=0.2086$, data not shown). Histamine was detected in 2 subjects with AS (subject 2, $0.032 \mu \mathrm{g} / \mathrm{ml}$; subject 7, $0.223 \mu \mathrm{g} / \mathrm{ml}$ ).

Microbiome analysis of CL care solution. The bacterial compositions of CL care solutions were assessed by 16S rDNA Illumina sequencing (Table SII). Diverse bacteria were detected in the CL care solutions even in the culture-negative samples. A BLAST search against the Greengenes database identified 98-304 OTUs/sample. As shown in Fig. 1A, 3 contamination patterns were observed in the CL care solutions of the subjects with AS by PCoA. Group CL-I (subjects 2, 7, 8 and 9) exhibited highly similar contamination patterns, which clearly differed from those of the healthy subjects. Groups CL-II 
A
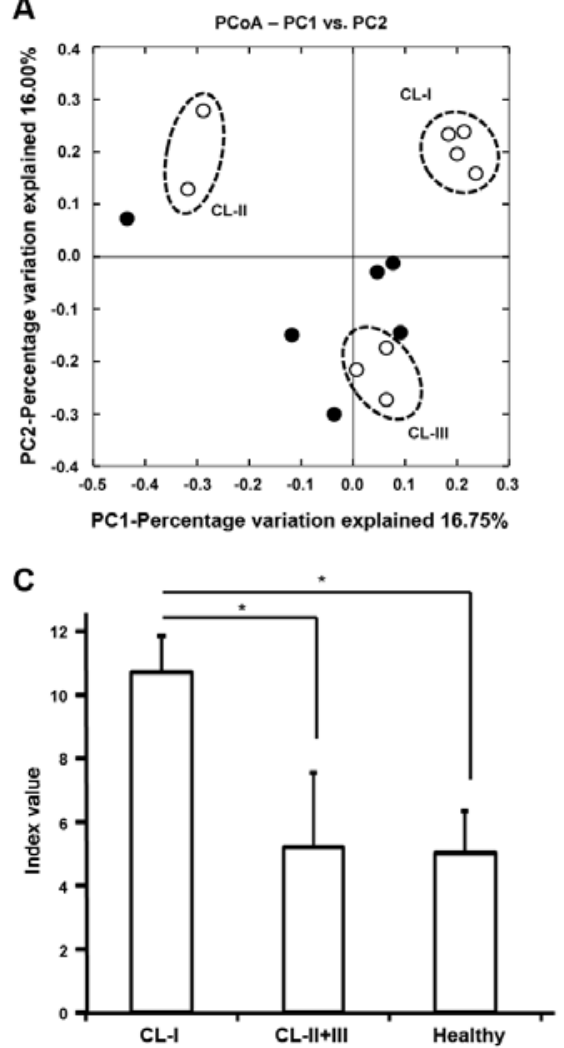

B

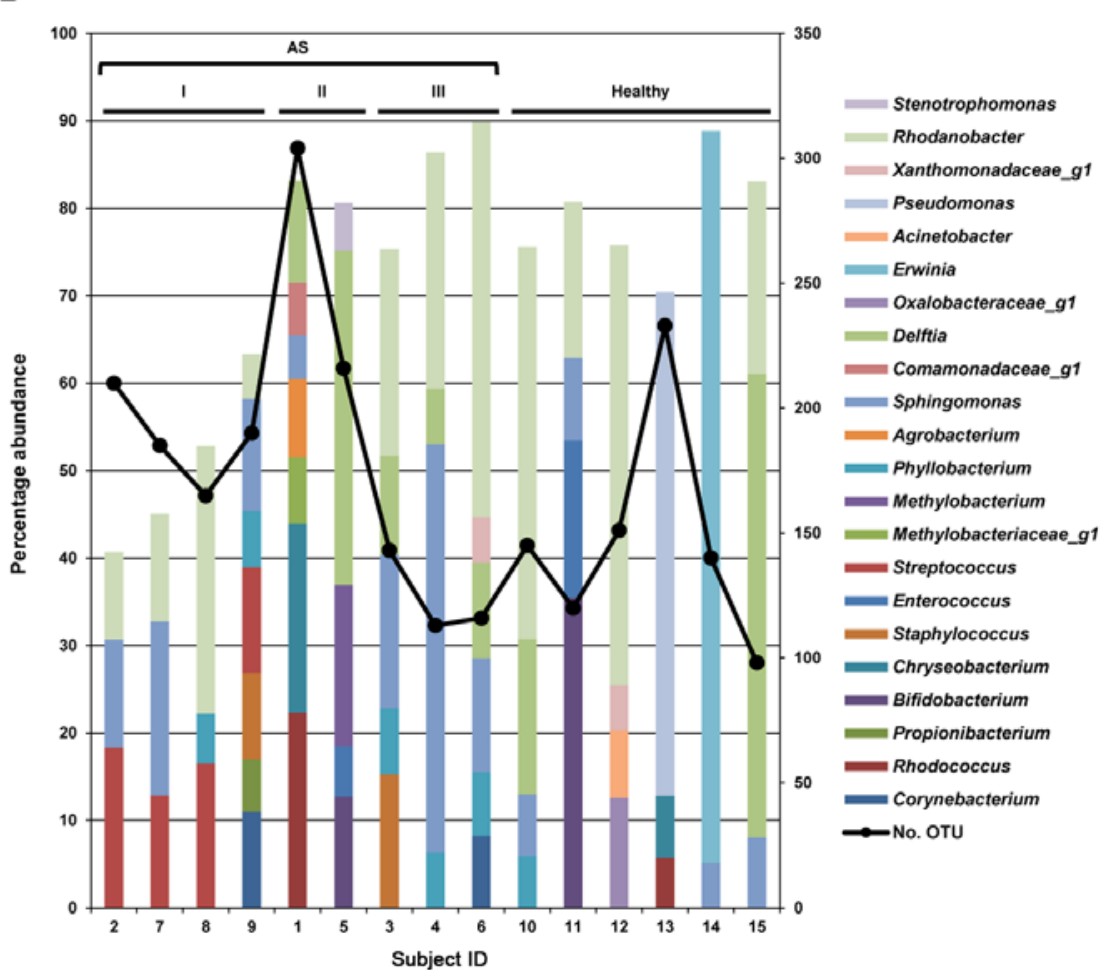

Figure 1. Microbiome analysis of contact lens care solutions. (A) PCoA of CL care solutions. Black dots denote healthy subjects, and white dots denote subjects with AS. Three contamination patterns were observed in the CL care solutions of AS subjects by PCoA. (B) Representative bacterial genera with $>5 \%$ abundance in each CL care solution. CL care solutions of the CL-I group uniquely contained Streptococcus (shown in magenta) as a dominant member of the contaminants. PCoA, principal coordinate analysis; CL, contact lens; AS, allergic symptoms; OTU, operational taxonomic unit; I, CL-I group; II, CL-II group; III, CL-III group. (C) Shannon-Weaver index. The Shannon-Weaver index of the CL-I group is significantly higher than that of the other subjects with AS or healthy subjects. ${ }^{*} \mathrm{P}<0.01$.

(subjects 1 and 5) and CL-III (subjects 3, 4 and 6) were not clearly separated from the healthy subjects. The abundance of Gram-positive bacteria in the microbiome of the CL care solutions used by the CL-I group was higher than that of the healthy subjects $(42.24 \pm 9.47 \%$ vs. $16.85 \pm 22.76 \%$ abundance) (Table SII). Fig. 1B shows the representative bacterial genera with $>5 \%$ abundance in each CL care solution. The CL care solutions of the CL-I group uniquely contained Streptococcus (shown in magenta) as a dominant member of the contaminants (12.1-18.3\% abundance). The total percentage of OTUs with $>5 \%$ abundance in the CL-I group was lower $(<65 \%)$ than that in the other subjects $(>70 \%)$, although the observed OTU number was relatively high (165-210). These findings indicate that the CL care solutions of the CL-I group contained a diverse minor population of bacteria. Correspondingly, the Shannon-Weaver index calculated from the abundance at the genus level was markedly higher in the CL-I group $(10.70 \pm 1.14)$ than in the other AS $(5.19 \pm 2.36)$ or healthy $(5.00 \pm 1.33)$ subjects $(\mathrm{P}<0.01)$ (Fig. 1C).

Microbiome analysis of tear fluid. The microbial composition in the tear fluid from the subjects with AS and healthy subjects was examined (Table SIII). The tear fluid from subject 1 was excluded from the analysis owing to its small volume. A total of 36-177 OTUs/sample wer detected. Of note, the tear microbiome analysis revealed similar trends to that of the $\mathrm{CL}$ care solutions. As shown in the PCoA plot, tear fluids from the subjects with AS clearly separated into groups resembling the microbiome patterns in CL care solutions (Fig. 2A). The representative bacterial genera with $>5 \%$ abundance in each tear fluid are shown in Fig. 2B. The total percentage of OTUs with $>5 \%$ abundance in the CL-I group was lower $(<50 \%)$ than that in other subjects $(>50 \%)$; however, the observed OTU number was relatively high (91-132). These results indicated that bacterial diversity in the tear fluids of the CL-I group was higher than that of the other subjects. Although Streptococcus was detected in all the tear fluids, apart from subject 5, its abundance in the CL-I group was significantly higher than that in the other subjects $(19.02 \pm 5.50$ vs. $3.08 \pm 3.35 \%, \mathrm{P}<0.01)$. To compare the microbial compositions in the CL care solutions and tear fluids, the Illumina sequences obtained from both types of samples were merged and PCoA was performed (Fig. 2C). Although the PCoA plots were clearly separated depending on the sample type, the plots denoting tear and CL care solution samples of the CL-I group were close to each other, as compared with the distance between plots denoting tear and CL care solution samples in subjects other than the CL-I group. This was due to the high abundance of Streptococcus in both types of samples in the CL-I group.

Detection of tear IgE reacting with Gram-positive cocci. The arrangement of bacterial antigens is presented in Fig. 3A. As 

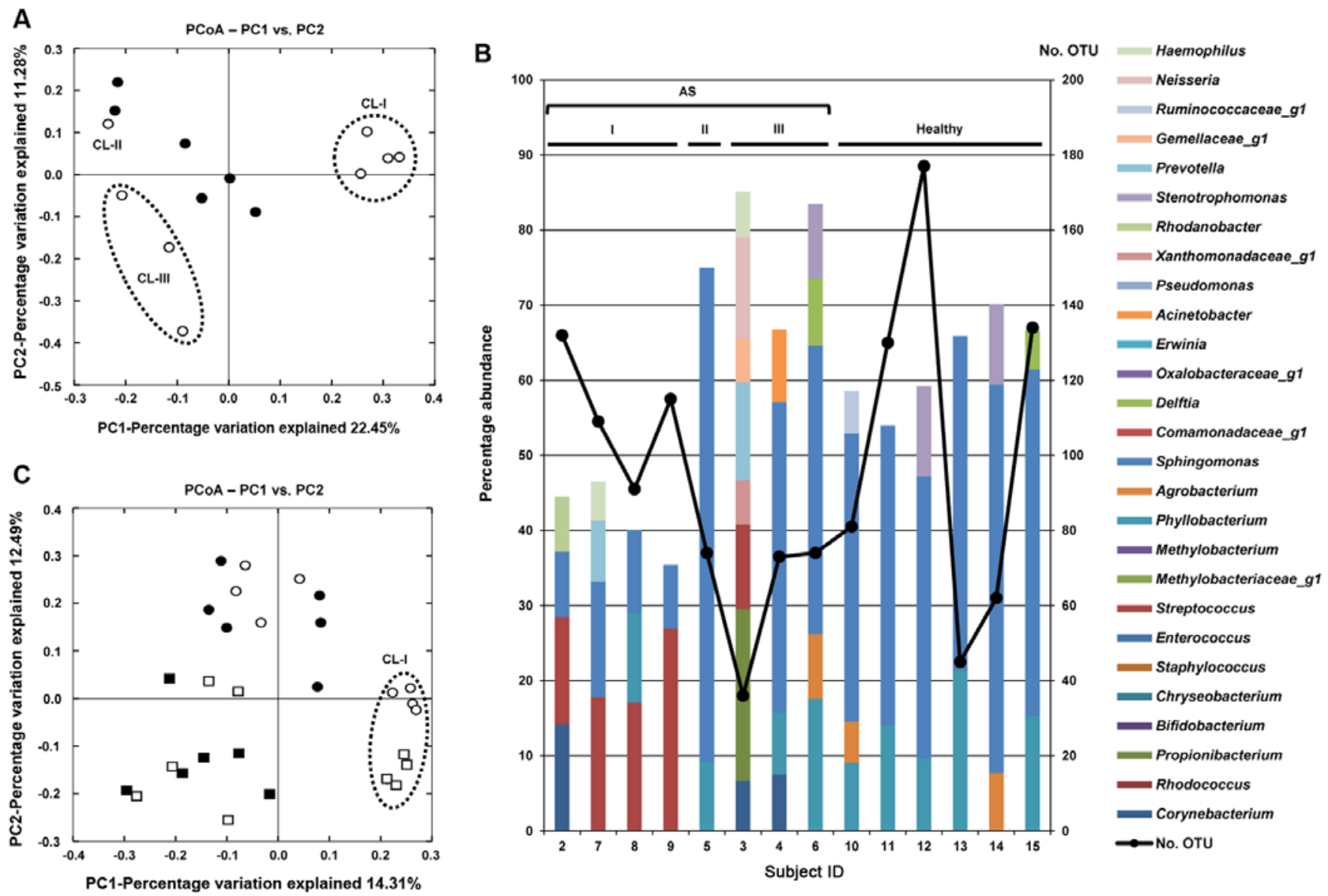

Figure 2. Microbiome analysis of tear fluids. (A) PCoA of tear fluids. Black dots denote healthy subjects, and white dots denote allergic symptoms (AS) subjects. Tear fluids from AS subjects clearly separated according to the grouping based on the microbiome patterns in contact lens (CL) care solutions. (B) Representative bacterial genera with $>5 \%$ abundance in each tear fluid. Proportion of Streptococcus in the tear fluid of the CL-I group was significantly higher than in the other subjects. (C) Comparison of the microbial compositions in CL care solutions and tear fluids. White circles denote tear fluids of subjects with AS, black circles denote tear fluids of healthy subjects, white squares denote CL care solutions of subjects with AS, and black squares denote CL care solutions of healthy subjects. The microbiomes in CL care solutions and tear fluids of the CL-I group were clustered closely by PCoA. PCoA, principal coordinate analysis; CL, contact lens; AS, allergic symptoms; OTU, operational taxonomic unit; I, CL-I group; II, CL-II group; III, CL-III group.

shown in Fig. 3B, IgE binding to the bacterial cell suspension of Streptococcus pneumoniae and Streptococcus tigurinus was detected in the tear fluids of 6 subjects with AS (100\%) as well as in 2 healthy subjects (100\%). IgE binding to Staphylococcus species was not present except for subject 11, in which IgE weakly reacted with concentrated Staphylococcus aureus cells. No signal was detected when the culture supernatants were used as antigens. Interestingly, IgE from human serum weakly reacted with Streptococcus tigurinus cells.

\section{Discussion}

A number of studies have reported that CL and the care accessories are contaminated with microorganisms not only in patients with infectious keratitis, but also in asymptomatic CL wearers (22-28). Sample cultivation has identified the contaminating microorganisms in the CL care accessories, and the most frequently isolated microorganisms are bacteria $(22,26,28)$. Among CL care accessories, the lens storage case is the most contaminated $(23,24)$, and there are reports that the contamination rate exceeds $80 \%(22,27)$. It is considered that lens storage cases are contaminated rapidly, followed by contamination spread to the CL and CL care solution bottles $(24,28)$. Shin et al analyzed the ocular surface microbiome of CL wearers using NGS technology and reported that CL wearing alters the microbiome (5). Considering the possibility that the source of CL contamination is the lens storage case $(24,28)$, it is reasonable to analyze the microbiome of the CL care solution in the lens storage case to identify the bacteria which migrate to the ocular surface by CL wearing.

In terms of the comparison of the subjects with AS and the healthy subjects, the present study first investigated both the CL care solutions in the lens storage cases and the tear fluids of the CL wearers by culture. It was found that the culture positive rate of the CL care solutions and the contaminant bacterial number did not differ significantly between the subjects with AS and the healthy subjects. As previously reported by Tagliaferri et al (4), this result suggests that CL care solutions of the subjects with AS are not always more contaminated by cultivatable bacteria than those from the healthy subjects. According to several articles that investigated lens storage case bacterial contamination, Staphylococcus species, Serratia species, and Pseudomonas species are frequently 
A

\begin{tabular}{|c|c|c|c|}
\hline $\begin{array}{c}\text { Human } \\
\text { IgE }\end{array}$ & $\begin{array}{c}\mathrm{SA} \\
5 \mathrm{OD} / \mathrm{ml}\end{array}$ & $\begin{array}{c}\mathrm{SA} \\
10 \mathrm{OD} / \mathrm{ml}\end{array}$ & $\mathrm{BHI}$ \\
\hline StrP sup & StrT sup & $\mathrm{SC}$ sup & SE sup \\
\hline $\begin{array}{c}\text { StrP } \\
5 \mathrm{OD} / \mathrm{ml}\end{array}$ & $\begin{array}{c}\mathrm{StrT} \\
5 \mathrm{OD} / \mathrm{ml}\end{array}$ & $\begin{array}{c}\mathrm{SC} \\
5 \mathrm{OD} / \mathrm{ml}\end{array}$ & $\begin{array}{c}\mathrm{SE} \\
5 \mathrm{OD} / \mathrm{ml}\end{array}$ \\
\hline
\end{tabular}

B

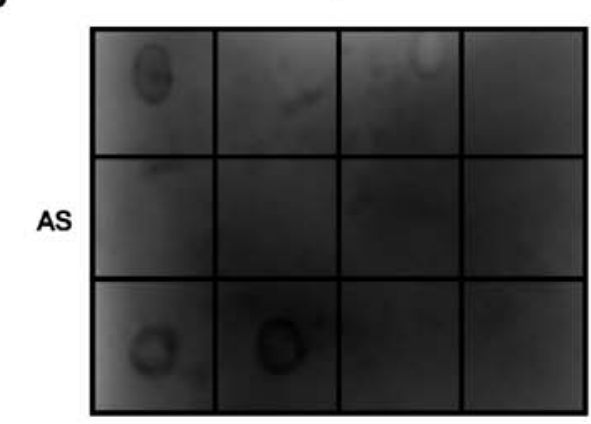

10

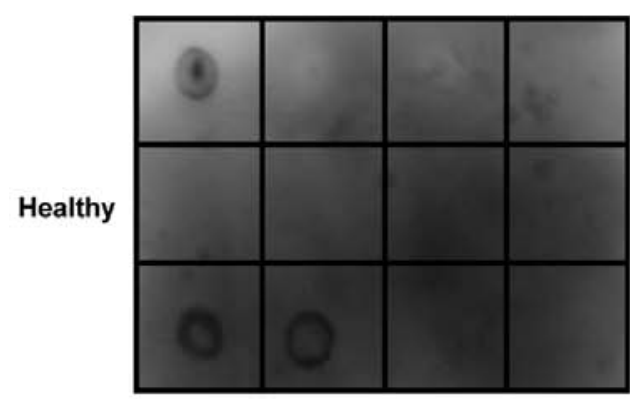

8

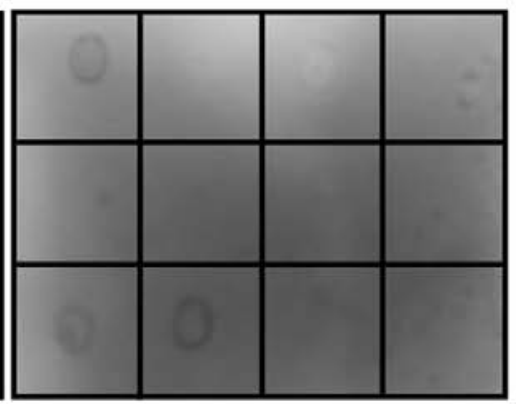

11

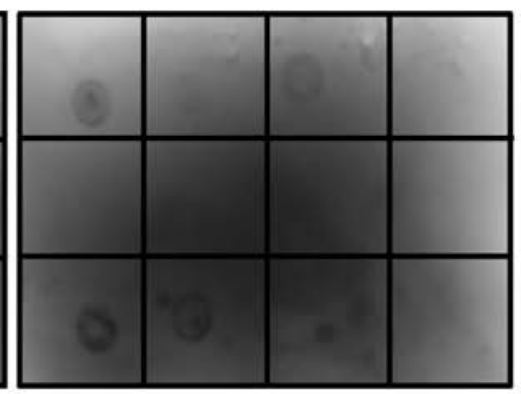

9

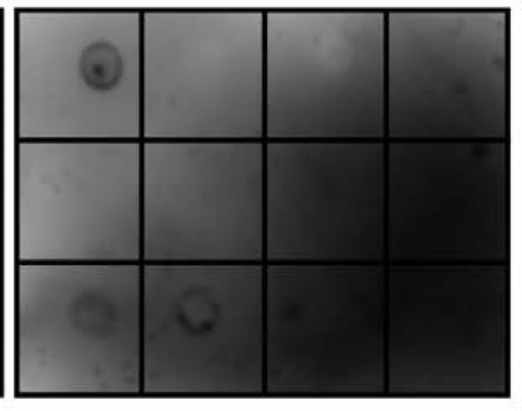

Human serum $\lg \mathrm{E}$

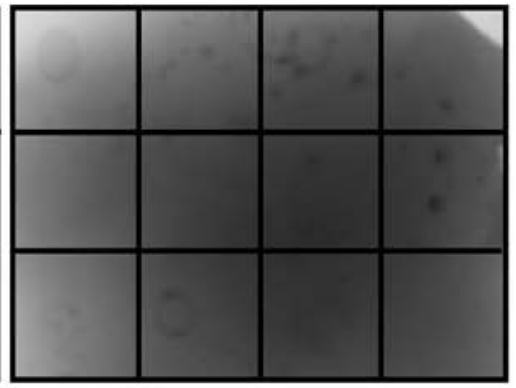

Figure 3. Western dot blot hybridization using tear fluids. (A) Arrangement of western dot blot hybridization. The nitrocellulose membrane was divided into 12 sections. Human IgE, positive control; SA, bacterial cell suspension of Staphylococcus (S.) aureus; BHI, brain heart infusion; negative control, StrP sup, culture supernatants of Streptococcus (Str.) pneumoniae; StrT sup, culture supernatants of Str. tigurinus; SC sup, culture supernatants of S. capitis; SE sup, culture supernatants of S. epidermidis; StrP, bacterial cell suspension of Str. pneumoniae; StrT, bacterial cell suspension of Str. tigurinus; SC, bacterial cell suspension of S. capitis; SE, bacterial cell suspension of S. epidermidis. (B) Images of nitrocellulose membrane. IgE binding to bacterial cell suspension of Str. pneumoniae and Str. tigurinus was detected in tear fluids from subjects with allergic symptoms (AS) as well as healthy subjects.

isolated $(23,24,26)$. The present study also mainly isolated Pseudomonas sp., Microbacterium sp. and Staphylococcus sp. (Table I). The contamination sources are considered to be fingers, eyelids and watery environments near the storage cases (25). However, there are a number of fastidious bacteria in the environment (6), and the percentage of environmental bacteria that can be grown in culture is only $1 \%$ (29). Attention should also be paid to the fact that cultivation can only detect viable bacteria. Not only viable bacteria, but also dead bacteria accumulating in the lens storage case may be involved in an allergic conjunctivitis onset (30). Therefore, meta-genomic analysis is more advantageous for investigating the microbiome of lens storage cases than conventional cultivation. With regard to the tear sample cultures, bacterial isolation failed for all but one of the subjects. It is reasonable to assume that a few microliters of the tear fluid contain only a minute number of bacteria, as the tear fluid plays a role in self-cleaning of the ocular surface using flow. Since it increases the probability of detection by amplifying DNA even in a specimen containing a small number of bacteria, genomic analysis is likely more useful than culture.

As a result of the present study of the microbiome in CL care solutions and tear fluids using NGS techniques, it was found that the bacteria contained in the CL care solutions and the tear fluids were more diverse than those detected by culture (Tables SII and SIII). CL wearing may affect the tear fluid microbiome by circulating the care solutions between the ocular surface and storage case, which is supported by the fact that the microbiome of tear fluids in subjects with AS was divided into 3 clusters, similar to that of CL care solutions (Fig. 2A). 
It was hypothesized that the contamination pattern of the CL care solution in each case varies depending on individual environmental differences. In fact, the microbiome of CL care solution did not exhibit a specific contamination pattern in healthy subjects in this study (Fig. 1A). PCoA revealed that the CL-I group in the subjects with AS was distinguished from the healthy subjects (Figs. 1A and 2A) and the histamine concentration in the tear fluids of the 2 subjects clustered in the CL-I group were elevated (Table I). The CL care solutions and the tear fluids of the CL-I group were found to be contaminated with a different population of bacteria as compared with other subjects (Figs. 1B, C and 2B). In particular, the proportion of Streptococcus were significantly higher (Figs. 1B and 2B), suggesting the possible involvement of Streptococcus in the etiology of at least certain subtypes of CL-associated AS. By contrast, in the CL-II and CL-III groups there was no significant difference in the microbiome of CL care solutions from the healthy subjects; therefore factors other than the change in the microbiome of CL care solutions may be related to AS in the CL-II and III groups. Alternatively, the lack of an elevation in the histamine concentration in the tear fluids of subjects in the CL-II and III groups suggests that symptoms in these subjects were not caused by an allergic response. Patients with corneal and/or conjunctival disorders may experience itching even without an allergic conjunctivitis $(31,32)$. The division of subjects with AS onto 3 clusters according to their microbiome PCoA plotting patterns may have occurred as the disease that caused itching in each group differed. However, whether the ocular surface microbiome would differ based on the disease causing the itching remains to be elucidated. Although dry eye and atopic dermatitis cases were excluded, the cause of the symptoms in subjects belonging to the CL-II and III groups cannot be specified, since all other diseases that can induce AS were not differentiated. Therefore, the reason for the 3 different microbiome PCOA plotting patterns cannot be specified in the present study.

$\mathrm{IgE}$ is an important chemical mediator in allergic diseases. CLPC, the allergic conjunctivitis associated with CL wear, is considered to be related to type I and type IV allergies (33). Studies have reported that IgE of the ocular surface of CLPC patients was significantly increased, compared with that of healthy CL wearers, owing to the involvement of type I allergy $(34,35)$. However, Zhao et al examined only the total IgE eluted from the CL and did not examine allergen-specific $\mathrm{IgE}$; thus, the antigen has not been clarified (34). Barishak et al examined the specific antigen of $\operatorname{IgE}$ in tear fluids of CLPC patients using radioallergosorbent test, and they detected $\operatorname{IgE}$ against house dust mites and cat epithelium only in one of those (35). In the present study, the western dot blotting method was used to identify bacteria-specific $\operatorname{IgE}$ in the tear fluids of subjects. In view of the results that a number of Gram-positive bacteria, particularly Firmicutes, were detected in the microbiome (Tables SII and SIII), the reactivity of the tear IgE against the Streptococcus and Staphylococcus was examined. IgE against Streptococcus was detected in tear fluid not only from subjects with AS, but also from healthy subjects (Fig. 3B), and the human serum IgE also responded to Streptococcus, suggesting that Streptococcus may have a strong influence on the human immune system. Bisgaard et al reported that the colonization of Streptococcus pneumoniae in the hypopharynx during the neonatal period was involved in the development of pediatric asthma (36). There is also a study reporting that the colonization rate of Streptococcus pneumoniae in the oropharynx is higher in asthma patients than in healthy subjects (37). Research published in recent years examining the airway microbiome using 16S rDNA analysis has revealed that early asymptomatic colonization with Streptococcus is a strong asthma predictor and the presence of Streptococcus sp. is related to severe asthma (38-41). Prior infection of the pharynx with Streptococcus is involved in the onset and exacerbation of psoriasis, an autoimmune skin disease. The molecular structure of streptococcal M-protein and keratinocyte protein are similar, so that streptococci-specific $\mathrm{T}$ cells are considered to cause a cross-reactivation with epidermal self-antigens (42-45). These suggests that Streptococcus is involved in the development and progression of several immune diseases, and it possibly plays a similarly important role in AS.

In the present study, the tear IgE response to Streptococcus pneumoniae and Streptococcus tigurinus was tested, both belonging to mitis group of streptococci (Fig. S1). Given that IgE did not react with the culture supernatant, but with the bacterial cells in the present study, it appears to react with common antigens of the mitis group of streptococci that are expressed on the bacterial surface but not with a substance produced and secreted by Streptococcus. Since the $\mathrm{IgE}$ reacting to streptococcal antigens is present in tear fluids of a wide range of individuals, repeated exposure to the mitis group of streptococci may elicit AS in certain populations.

There are several limitations to the present study. First, the sample size was small. Patients were recruited who had not suffered extensively from wearing contact lenses in their daily life. Therefore, it was difficult to collect a large number of candidates without providing incentives. Consequently, there was a marked sex difference, with 12 females and 3 males. Although there have been several reports of no sex differences regarding the rate of contamination of $\mathrm{CL}$ care accessories $(46,47)$, a biased sex ratio may still be a confounding factor in the current microbiome analysis. CLPC is more common in long-term CL wearers. However, in the present study, all subjects were in their late teens or early 20s, and were using CLs for $<5$ years; thus, CLPC was mild in all subjects. If a similar microbiome study could be conducted by dividing a large number of subjects based on short- and long-term CL wear experience, then new findings may be obtained. Although the sample size was tolerable in the present study, a larger sample size would still be more reliable. Second, itching can also occur in eye diseases other than allergies. Therefore, the results of the present study do not suggest that Streptococcus spp. are associated with allergic eye diseases, but only that they are associated with their symptoms. Third, as the antigens have not been comprehensively examined, the possibility that there may be an antigen related to AS other than Streptococcus, such as certain aeroallergens remains. Fourth, from the results of the present study, it was undetermined whether AS developed due to the high proportion of Streptococcus in the CL care solution and tear microbiome or if the microbiome alteration following allergy development resulted in the high proportion of Streptococcus in these samples. Finally, 
in specimens with a low bacterial volume, the results may be affected by contamination that can occur in all steps from sample collection to DNA manipulation, although it is impossible to completely avoid bacterial contamination from all experimental processes. If the experimental process is the same in all samples, the contamination level would be expected to be the same. Further studies with large numbers of allergic conjunctivitis subjects, diagnosed by serum IgE measurement and conjunctival scraping to detect eosinophils and lacking bacterial contamination of the ocular surface microbiome are required to resolve these limitations. PCoA with an increased sample size may provide new trends for the species involved in allergic conjunctivitis. If the presence of specific IgE, in tears and serum, can be confirmed for that species, the identification of the ones involved in allergic conjunctivitis may then be possible.

In conclusion, in the present study, the CL care solutions remaining in lens storage cases and tear fluids of some CL wearers who suffer from AS exhibited a significantly higher relative level of Streptococcus, and the IgE reacting to streptococcal antigens was present in tear fluids. This suggests an association between Streptococcus contamination of the lens storage case and AS associated with CL wear. However, further studies are required to determine one of the true antigens of AS associated with CL wear.

\section{Acknowledgements}

The authors would like to thank Mr. Yu Noguchi and $\mathrm{Mr}$. Takuya Taoka, Tokushima University, School of Medicine, for providing assistance with collecting the CL storage cases.

\section{Funding}

HE received grant funding from Pfizer Japan Inc. YS received grant funding from Pfizer Japan Inc. and Daiichi Sankyo Co Ltd.

\section{Availability of data and materials}

The datasets used and/or analyzed during the current study are available from the corresponding author on reasonable request. Sequence data have been deposited in the DDBJ database (accession nos. DRA010383: https://ddbj.nig.ac.jp/DRASearch/ submission?acc=DRA010383; PRJDB10068: https://ddbj.nig. ac.jp/BPSearch/bioproject?acc=PRJDB10068; SAMD00232908SAMD00232922: https://ddbj.nig.ac.jp/DRASearch/experiment? acc=DRX224997, https://ddbj.nig.ac.jp/DRASearch/experiment? acc=DRX224998, https://ddbj.nig.ac.jp/DRASearch/experiment? acc $=$ DR X224999, https://ddbj.nig.ac.jp/DRASearch/ experiment?acc=DRX225000, https://ddbj.nig.ac.jp/DRASearch/ experiment? acc=DRX225001,https://ddbj.nig.ac.jp/DRASearch/ experiment?acc=DRX225002, https://ddbj.nig.ac.jp/DRASearch/ experiment?acc=DRX225003, https://ddbj.nig.ac.jp/DRASearch/ experiment?acc=DRX225004, https://ddbj.nig.ac.jp/DRASearch/ experiment?acc=DRX225005, https://ddbj.nig.ac.jp/DRASearch/ experiment?acc=DRX225006, https://ddbj.nig.ac.jp/DRASearch/ experiment?acc=DRX225007, https://ddbj.nig.ac.jp/DRASearch/ experiment?acc=DRX225008, https://ddbj.nig.ac.jp/DRASearch/
experiment?acc=DRX225009, https://ddbj.nig.ac.jp/DRASearch/ experiment?acc=DRX225010, https://ddbj.nig.ac.jp/DRASearch/ experiment?acc $=\mathrm{DRX} 225011)$.

\section{Authors' contributions}

HE and TK conceived the study, established the methodology, and administered the projects. FH, HE, HNI, AT and HY curated the data. TK and HNI analyzed the data and supervised the study. FH, HE, and TK validated the results. FH, $\mathrm{HE}$, and TK wrote the original draft of the manuscript. HE, TK, YS and SK conceived and supervised the study, reviewed and edited the original draft. All authors read and approved the final manuscript.

\section{Ethics approval and consent to participate}

The present study followed the tenets of the Declaration of Helsinki and was approved by the institutional review board of Kindai University Faculty of Medicine (Approval no. 28-137). Informed consent was obtained from all subjects after explanation of the nature and possible consequences of the study.

\section{Patient consent for publication}

Not applicable.

\section{Competing interests}

The authors declare that they have no competing interests.

\section{References}

1. Skotnitsky CC, Naduvilath TJ, Sweeney DF and Sankaridurg PR: Two presentations of contact lens-induced papillary conjunctivitis (CLPC) in hydrogel lens wear: Local and general. Optom Vis Sci 83: 27-36, 2006.

2. Solomon A: Allergic manifestations of contact lens wearing. Curr Opin Allergy Clin Immunol 16: 492-497, 2016.

3. Tan ME, Demirci G, Pearce D, Jalbert I, Sankaridurg P and Willcox MD: Contact lens-induced papillary conjunctivitis is associated with increased albumin deposits on extended wear hydrogel lenses. Adv Exp Med Biol 506: 951-955, 2002.

4. Tagliaferri A, Love TE and Szczotka-Flynn LB: Risk factors for contact lens-induced papillary conjunctivitis associated with silicone hydrogel contact lens wear. Eye Contact Lens 40: 117-122, 2014.

5. Shin H, Price K, Albert L, Dodick J, Park L and Dominguez-Bello MG: Changes in the eye microbiota associated with contact lens wearing. MBio 7: e00198, 2016.

6. Amann RI, Ludwig W and Schleifer KH: Phylogenetic identification and in situ detection of indivisual microbial cells without cultivation. Microbiol Rev 59: 143-169, 1995.

7. Human Microbiome Project Consortium: Structure, function and diversity of the healthy human microbiome. Nature 486: 207-214, 2012.

8. Ahmed S, Macfarlane GT, Fite A, McBain AJ, Gilbert P and Macfarlane S: Mucosa-associated bacterial diversity in relation to human terminal ileum and colonic biopsy samples. Appl Environ Microbiol 73: 7435-7442, 2007.

9. Grice EA, Kong HH, Renaud G, Young AC, NISC Comparative Sequencing Program; Bouffard GG, Blakesley RW, Wolfsberg TG, Turner ML and Segre JA: A diversity profile of the human skin microbiota. Genome Res 18: 1043-1050, 2008.

10. Dong Q, Brulc JM, Iovieno A, Bates B, Garoutte A, Miller D, Revanna KV, Gao X, Antonopoulos DA, Slepak VZ and Shestopalov VI: Diversity of bacteria at healthy human conjunctiva. Invest Ophthalmol Vis Sci 52: 5408-5413, 2011 
11. Eguchi H, Hotta F, Kuwahara T, Imaohji H, Miyazaki C Hirose M, Kusaka S, Fukuda M and Shimomura Y: Diagnostic approach to ocular infections using various techniques from conventional culture to next-generation sequencing analysis. Cornea 36 (Suppl 1): S46-S52, 2017.

12. Huang Y, Yang B and Li W: Defining the normal core microbiome of conjunctival microbial communities. Clin Microbiol Infect 22: 643.e7-643.e12, 2016.

13. Bakhtiar SM, LeBlanc JG, Salvucci E, Ali A, Martin R, Langella P, Chatel JM, Miyoshi A, Bermúdez-Humarán LG and Azevedo V: Implications of the human microbiome in inflammatory bowel diseases. FEMS Microbiol Lett 342: 10-17, 2013.

14. Turnbaugh PJ, Ley RE, Mahowald MA, Magrini V, Mardis ER and Gordon JI: An obesity-associated gut microbiome with increased capacity for energy harvest. Nature 444: 1027-1031, 2006.

15. Chiacchierini E, Restuccia D and Vinci G: Evaluation of two different extraction methods for chromatographic determination of bioactive amines in tomato products. Talanta 69: 548-555, 2006

16. Morita H, Kuwahara T, Ohshima K, Sasamoto H, Itoh K, Hattori M, Hayashi T and Takami H: An improved DNA isolation method for metagenomic analysis of the microbial flora of the human intestine. Microbes Environ 22: 214-222, 2007.

17. Klindworth A, Pruesse E, Schweer T, Peplies J, Quast C, Horn M and Glöckner FO: Evaluation of general 16S ribosomal RNA gene PCR primers for classical and next-generation sequencing-based diversity studies. Nucleic Acids Res 41: e1, 2013.

18. Aronesty E: Comparison of sequencing utility programs. Open Bioinformatics J 7: 1-8, 2013

19. Navas-Molina JA, Peralta-Sánchez JM, González A, McMurdie PJ, Vázquez-Baeza Y, Xu Z, Ursell LK, Lauber C, Zhou $\mathrm{H}$, Song SJ, et al: Advancing our understanding of the human microbiome using QIIME. Methods Enzymol 531: 371-444, 2013

20. Edgar RC: Search and clustering orders of magnitude faster than BLAST. Bioinformatics 26: 2460-2461, 2010.

21. Kanda Y: Investigation of the freely available easy-to-use software 'EZR' for medical statistics. Bone Marrow Transplant 48: 452-458, 2013

22. Dantam J, McCanna DJ, Subbaraman LN, Papinski D, Lakkis C, Mirza A, Berntsen DA, Morgan P, Nichols JJ and Jones LW: Microbial contamination of contact lens storage cases during daily wear use. Optom Vis Sci 93: 925-932, 2016.

23. Boost MV and Cho P: Microbial flora of tears of orthokeratology patients, and microbial contamination of contact lenses and contact lens accessories. Optom Vis Sci 82: 451-458, 2005.

24. Yung MS, Boost M, Cho P and Yap M: Microbial contamination of contact lenses and lens care accessories of soft contact lens wearers (university students) in Hong Kong. Ophthalmic Physiol Opt 27: 11-21, 2007.

25. Willcox MD, Power KN, Stapleton F, Leitch C, Harmis N and Sweeney DF: Potential sources of bacteria that are isolated from contact lenses during wear. Optom Vis Sci 74: 1030-1038, 1997.

26. Wu YT, Zhu H, Harmis NY, Iskandar SY, Willcox M and Stapleton F: Profile and frequency of microbial contamination of contact lens cases. Optom Vis Sci 87: E152-E158, 2010.

27. Willcox MD, Carnt N, Diec J, Naduvilath T, Evans V, Stapleton F, Iskandar S, Harmis N, de la Jara PL and Holden BA: Contact lens case contamination during daily wear of silicone hydrogels. Optom Vis Sci 87: 456-464, 2010.

28. McLaughlin-Borlace L, Stapleton F, Matheson M and Dart JK: Bacterial biofilm on contact lenses and lens storage cases in wearers with microbial keratitis. J Appl Microbiol 84: 827-838, 1998.

29. Staley JT and Konopka A: Measurement of in situ activities of nonphotosynthetic microorganisms in aquatic and terrestrial habitats. Annu Rev Microbiol 39: 321-346, 1985.

30. Eguchi H, Hotta F, Kuwahara T, Nakayama-Imaohji H, Kusaka S and Shimomura Y: Acute keratoconjunctivitis due to contamination of contact lens care solution with histamine-producing Raoultella species. A case report. Medicine (Baltimore) 96: e9310, 2017.
31. Nichols KK: Patient-reported symptoms in dry eye disease. Ocul Surf 4: 137-145, 2006.

32. Belmonte C: Pain, dryness, and itch sensations in eye surface disorders are defined by a balance between inflammation and sensory nerve injury. Cornea 38 (Suppl 1): S11-S24, 2019.

33. Stapleton F, Stretton S, Sankaridurg PR, Chandoha H and Shovlin J: Hypersensitivity responses and contact lens wear. Cont Lens Anterior Eye 26: 57-69, 2003.

34. Zhao Z, Fu H, Skotnitsky CC, Sankaridurg PR and Willcox MD: IgE antibody on worn highly oxygen-permeable silicone hydrogel contact lenses from patients with contact lens-induced papillary conjunctivitis (CLPC). Eye Contact Lens 34: 117-121, 2008.

35. Barishak Y, Zavaro A, Samra Z and Sompolinsky D: An immunological study of papillary conjunctivitis due to contact lenses. Curr Eye Res 3: 1161-1168, 1984.

36. Bisgaard H, Hermansen MN, Buchvald F, Loland L, Halkjaer LB, Bønnelykke K, Brasholt M, Heltberg A, Vissing NH, Thorsen SV, et al: Childhood asthma after bacterial colonization of the airway in neonates. N Engl J Med 357: 1487-1495, 2007.

37. Jounio U, Juvonen R, Bloigu A, Silvennoinen-Kassinen S, Kaijalainen T, Kauma H, Peitso A, Saukkoriipi A, Vainio O, Harju $\mathrm{T}$ and Leinonen $\mathrm{M}$ : Pneumococcal carriage is more common in asthmatic than in non-asthmatic young men. Clin Respir J 4: 222-229, 2010

38. Teo SM, Mok D, Pham K, Kusel M, Serralha M, Troy N, Holt BJ, Hales BJ, Walker ML, Hollams E, et al: The infant airway microbiome in health and disease impacts later asthma development. Cell Host Microbe 17: 704-715, 2015.

39. Zhang Q, Cox M, Liang Z, Brinkmann F, Cardenas PA, Duff R, Bhavsar P, Cookson W, Moffatt M and Chung KF: Airway microbiota in severe asthma and relationship to asthma severity and phenotypes. PLoS One 11: e0152724, 2016.

40. Kloepfer KM,Lee WM,Pappas TE, Kang TJ, Vrtis RF,Evans MD, Gangnon RE, Bochkov YA, Jackson DJ, Lemanske RF Jr, et al: Detection of pathogenic bacteria during rhinovirus infection is associated with increased respiratory symptoms and exacerbations of asthma. J Allergy Clin Immunol 133: 1301-1307, e1-e3, 2014.

41. Green BJ, Wiriyachaiporn S, Grainge C, Rogers GB, Kehagia V, Lau L, Carroll MP, Bruce KD and Howarth PH: Potentially pathogenic airway bacteria and neutrophilic inflammation in treatment resistant severe asthma. PLoS One 9: e100645, 2014.

42. Weisenseel $P$ and Prinz JC: Incidental detection of $S$. pyogenes-DNA in psoriatic skin by PCR. Arch Dermatol Res 296: 573-576, 2005.

43. Valdimarsson H, Thorleifsdottir RH, Sigurdardottir SL, Gudjonsson JE and Johnston A: Psoriasis-as an autoimmune disease caused by molecular mimicry. Trends Immunol 30: 494-501, 2009.

44. Prinz JC: Psoriasis vulgaris-a sterile antibacterial skin reaction mediated by cross-reactive T cells? An immunological view of the pathophysiology of psoriasis. Clin Exp Dermatol 26: 326-332, 2001

45. Gudjonsson JE, Thorarinsson AM, Sigurgeirsson B, Kristinsson KG and Valdimarsson H: Streptococcal throat infections and exacerbation of chronic plaque psoriasis: A prospective study. Br J Dermatol 149: 530-534, 2003.

46. Gray TB, Cursons RT, Sherwan JF and Rose PR: Acanthamoeba, bacterial, and fungal contamination of contact lens storage cases. Br J Ophthalmol 79: 601-605, 1995.

47. Devonshire P, Munro FA, Abernethy C and Clark BJ: Microbial contamination of contact lens cases in the west of Scotland. $\mathrm{Br}$ J Ophthalmol 77: 41-45, 1993.

This work is licensed under a Creative Commons Attribution-NonCommercial-NoDerivatives 4.0 International (CC BY-NC-ND 4.0) License. 